\title{
Mamíferos terrestres de médio e grande porte em uma área de reserva legal na região Centro-Oeste de Minas Gerais, Brasil
}

\author{
Alysson R. Fonseca ${ }^{1 *}$ \\ Gabriele A. Silva ${ }^{2}$ \\ ${ }^{1}$ Universidade do Estado de Minas Gerais \\ Avenida Paraná, 3.001, Jardim Belvedere, CEP 35.501-170, Divinópolis - MG, Brasil \\ ${ }^{2}$ Universidade Federal de Uberlândia, Instituto de Biologia, \\ Rua Ceará, s/nº, CEP 38.405-315, Uberlândia - MG, Brasil \\ * Autor para correspondência \\ arodrigofonseca@hotmail.com
}

Submetido em 06/03/2019

Aceito para publicação em 14/08/2019

\section{Resumo}

As Reservas Legais (RL) são áreas localizadas dentro de propriedades rurais e contribuem para a manutenção da diversidade de espécies de mamíferos, bem como para a movimentação de espécies entre os fragmentos de vegetação. Este trabalho teve como objetivo quantificar a riqueza e analisar a frequência de ocorrência de mamíferos terrestres de médio e grande porte em uma área de RL de 76,5 ha, no município de Pedra do Indaiá, Minas Gerais, Brasil. O período de amostragem foi de 28 meses, com a utilização dos seguintes métodos de coleta: observação direta, indireta e armadilha fotográfica. Foram registradas 25 espécies, distribuídas em sete ordens e 14 famílias. As ordens mais representativas foram Carnivora, Cingulata e Rodentia, e as menos representativas foram Lagomorpha e Didelphimorphia. Das 25 espécies registradas, seis encontram-se ameaçadas de extinção na lista de espécies da fauna ameaçada no estado de Minas Gerais; e quatro, na lista de espécies da fauna ameaçada de extinção no Brasil. A presença de animais em alguma categoria de ameaça sugere a necessidade de manutenção da RL, bem como de remanescentes de vegetação nativa na região a fim de manter a qualidade do habitat necessária à persistência das populações e os serviços ecossistêmicos prestados por ela.

Palavras-chave: Cerrado; Conservação; Espécies ameaçadas; Levantamento

\section{Abstract}

Medium- and large-sized terrestrial mammals in a nature reserve area in central-western Minas Gerais State. Brazilian legal reserves (LRs) are preservation areas located on private land and contribute to the maintenance of mammal species diversity and their movement patterns between vegetation fragments. This study sought to quantify the richness and analyze the occurrence frequency of the medium- and large-sized terrestrial mammals of a 76.5 ha LR in the municipality of Pedra do Indaiá in Minas Gerais State, Brazil. Data collection lasted for 28 months and was based on the following sampling methods: direct and indirect observation and camera trapping. We registered 25 species from seven orders and 14 families. The most represented orders were Carnivora, Cingulata and Rodentia, while Lagomorpha and Didelphimorphia were the least recorded orders. Of the 25 recorded species, six are cited as threatened with extinction on the Minas Gerais Red List and four are 
cited in the Brazil Red Book of Threatened Fauna. The occurrence of threatened animal species suggests the need to maintain the LR and the native vegetation remnants in order to keep the habitat quality necessary for the persistence of populations and their ecosystem services.

Key words: Cerrado; Conservation; Threatened species; Survey

\section{Introdução}

Os mamíferos desempenham um papel fundamental na manutenção e no equilíbrio dos ecossistemas (ABREU JR; KÖHLER, 2009; GALETTI et al., 2010; MACHADO et al., 2017). As espécies frugívoras e/ ou herbívoras auxiliam na manutenção de espécies vegetais, por meio da dispersão de sementes, enquanto os carnívoros regulam as populações de herbívoros e frugívoros (TONHASCA JR., 2005; DUPRAT; ANDRIOLO, 2011). Aliado a isso, algumas espécies podem atuar como polinizadoras (SAZIMA et al., 1982) ou indicadoras ambientais, refletindo a qualidade do ambiente onde ocorrem (MAZZOLLI, 2006).

Apesar da importância da mastofauna para manutenção do ecossistema, estima-se que apenas $21 \%$ da superfície terrestre mantém sua fauna de mamíferos completa (MORRISON et al., 2007). Sugerem-se que haverá novas extinções de mamíferos (JOHNSON et al., 2017), sendo as espécies de maior porte as mais predispostas a declinar (CARDILLO et al., 2005). No Brasil, a perda e alteração do habitat são as principais ameaças aos mamíferos terrestres, que ainda sofrem com a pressão de caça (COSTA et al., 2005).

Estudos envolvendo riqueza, diversidade, abundância, frequência e uso do habitat têm se tornado cada vez mais frequentes (SANTOS-FILHO; SILVA, 2002; ROCHA; DALPONTE, 2006; DOTTA; VERDADE, 2007; ANTUNES; ESTON, 2009; OLIVEIRA et al., 2009; BOCCHIGLIERI et al., 2010; ALVES et al., 2012; BROCARDO; CANDIDO JUNIOR, 2012; PINHEIRO et al., 2017). Esses tipos de trabalhos são considerados ferramentas imprescindíveis para nortear ações e políticas de conservação, como por exemplo, medidas para criação e gestão áreas protegidas (PARDINI et al., 2006).

As Reservas Legais (RL) são áreas localizadas dentro de propriedades rurais que têm o objetivo de preservar parte dos recursos naturais existentes, bem como assegurar seu uso econômico de maneira sustentável e auxiliar na manutenção dos processos ecológicos (BRASIL, 2012). São, portanto, fragmentos mantidos por força de lei e se encontram presentes devido ao Código Florestal em vigência (Lei $\mathrm{n}^{\circ}$ 12.651/2012) (RIBEIRO; MELO, 2013). No entanto, discussões sobre as extensões e regras das RL durante a elaboração do Código Florestal vigente foram polêmicas e pesquisadores relataram graves prejuízos ao patrimônio biológico e genético. Essa preocupação se deve à redução da área de RL e sua junção com áreas de preservação permanente, bem como sua recomposição com espécies exóticas, fazendo com que haja redução na biodiversidade (METZGER, 2010; RORIZ; FEARNSIDE, 2015).

As RL são fundamentais para complementar as Unidades de Conservação, pois contribuem para manutenção da diversidade de espécies, aumentando a área de habitat disponível e criando paisagens com maior conectividade, e, ainda, facilitam a movimentação de espécies de maior porte entre os remanescentes de vegetação, permitindo o fluxo de animais (GALETTI et al., 2010). Assim, estudos que reforcem a necessidade de conservação desses fragmentos (RL) podem ajudar na criação de instrumentos que visem reduzir o impacto sobre a biodiversidade associada a esses locais, bem como os serviços ecossistêmicos prestados por ela. $\mathrm{O}$ objetivo do presente trabalho foi quantificar a riqueza e analisar a frequência de ocorrência de mamíferos terrestres de médio e grande porte em uma área de RL no município de Pedra do Indaiá, Minas Gerais, Brasil.

\section{Material e Métodos}

\section{Área de estudo}

O estudo foi realizado no município de Pedra do Indaiá, localizado na região Centro-Oeste do estado de 
Minas Gerais, Brasil (IBGE, 2010). O clima da região é caracterizado por invernos secos e verões chuvosos, com temperatura e precipitação média anual de $20,7^{\circ} \mathrm{C}$ e $1420 \mathrm{~mm}$, respectivamente. O município está inserido em uma região de transição entre o domínio Cerrado e Mata Atlântica (ZEE/MG, 2009).

As amostragens foram realizadas em uma área de RL (2016'18'S; 459'45'W) (Figura 1) de 76,5 ha instituída no sistema de condomínio, pertencente a cinco propriedades rurais. A vegetação local é caracterizada pela presença de formações de cerrado stricto sensu e mata de galeria, em diferentes graus de regeneração. A área encontra-se, aproximadamente, a $800 \mathrm{~m}$ do rio Lambari, que pertence à bacia do rio São Francisco. Nela existem cerca de três nascentes e uma área brejosa, que dão origem a cursos d'água distintos e que apresentam a mata ciliar preservada em maior parte de seu percurso.

\section{Coleta de dados}

O período de amostragem foi de 28 meses (janeiro/2013 a abril/2015), com a utilização dos seguintes métodos de coleta:

1. Direto: detecção visual durante censos crepusculares e noturnos (das 5 às $8 \mathrm{~h}$ e das 17 às $20 \mathrm{~h}$, respectivamente) realizados a pé com velocidade média de $1,5 \mathrm{~km} / \mathrm{h}$ em um transecto de $1,3 \mathrm{~km}$ localizado dentro da RL (Figura 1), uma vez ao mês, totalizando $168 \mathrm{~h}$ de observação.

2. Indireto: identificação de pegadas, vocalização e fezes. As observações foram realizadas consecutivamente à observação direta citada anteriormente, no mesmo transecto e horário.

3. Armadilhas fotográficas: utilização de três câmeras (modelos Moutrie ${ }^{\circledR}$ A5 e modelo Bushnell Trophy

FIGURA 1: Localização geográfica da Reserva Legal no município de Pedra do Indaiá, Minas Gerais, Brasil.

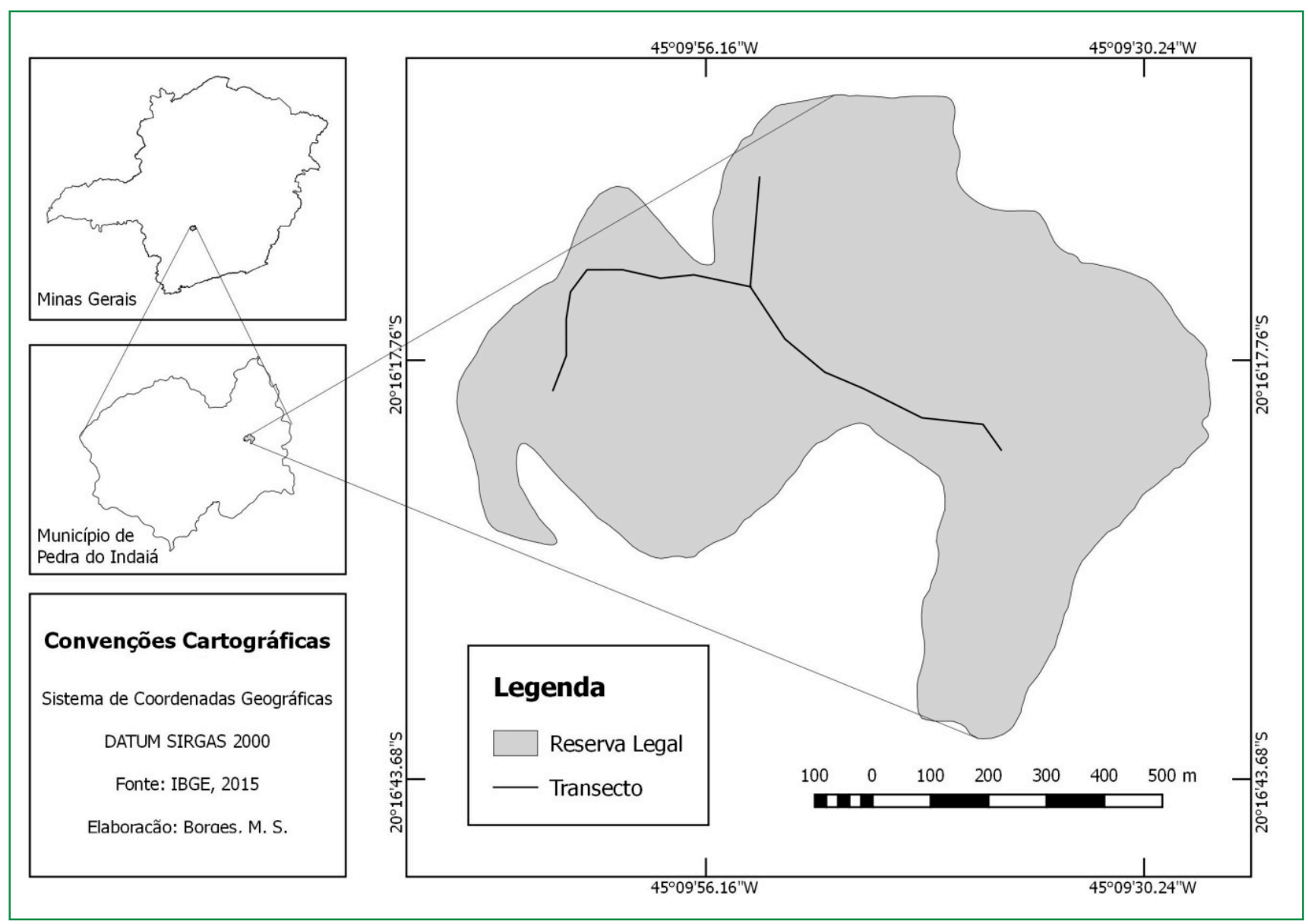


Cam ${ }^{\circledR}$ HD) a uma altura de $40 \mathrm{~cm}$ do chão, durante três dias por semana, correspondendo a 1.008 armadilhas-dia de esforço amostral.

Foram considerados mamíferos de médio e grande porte aqueles com peso corporal acima de um quilo quando adultos (PAGLIA et al., 2012). Embora Callithrix penicillata seja de pequeno porte, foi incluído neste estudo por ser seguramente identificado na área amostrada.

\section{Análise dos dados}

A identificação das espécies foi realizada com base em Oliveira e Cassaro (1999), Borges e Tomás (2004); Canevari e Vaccaro (2007) e Becker e Dalponte (2013); e a nomenclatura seguiu Paglia et al. (2012). A curva de acumulação de espécies foi calculada utilizando o estimador Jackknife de primeira ordem através do programa EstimateS 9.1 (COLWELL, 2013). A probabilidade de ocorrência foi calculada utilizando o índice de frequência de ocorrência (LINSDALE; ROGERS, 1937), representada pela seguinte equação:

$$
\mathrm{FO}=\mathrm{DR} / \mathrm{DO}^{*} 100
$$

Onde:

$\mathrm{FO}=$ frequência de ocorrência

$\mathrm{DR}=\mathrm{n}^{\mathrm{o}}$ de dias em que a espécie foi registrada

$\mathrm{DO}=\mathrm{n}^{\mathrm{o}}$ de dias de observação.
Em seguida, as espécies foram agrupadas em classes de frequência, onde: (a) muito abundante (81 a 100); (b) abundante (61-80); (c) frequente (41-60), (d) ocasional (21-40), (e) rara (1-20) e muito rara $(<1)$ (LINSDALE; ROGERS, 1937). Para todas as espécies registradas foi verificada a categoria de ameaçada de acordo com COPAM (2010) e da Portaria MMA 444 (MMA, 2014).

\section{Resultados}

Foram registradas 25 espécies de mamíferos terrestres de médio e grande porte, distribuídas em sete ordens e 14 famílias (Tabela 1). As ordens mais representativas foram Carnivora (52\%), Cingulata e Rodentia ( $12 \%$ cada), e as menos representativas foram Lagomorpha e Didelphimorphia (4\% cada). Os registros obtidos através das armadilhas fotográficas possibilitaram a identificação de 22 espécies, e o método de busca direto e/ou indireto, 14 espécies (Tabela 1).

A curva de acumulação de espécies apresentou um formato ascendente, com riqueza estimada em 30 espécies (Figura 2). Quanto às classes de frequência, $76 \%$ das espécies registradas foram consideradas raras, $12 \%$ muito raras, $8 \%$ ocasionais e $4 \%$ frequentes. As espécies muito raras registradas foram $L$. longicaudis, $P$. yagouaroundi e T. tetradactyla, e a única espécie considerada frequente foi $M$. tridactyla

TABELA 1: Espécies de mamíferos terrestres de médio e grande porte registrados em uma área de Reserva Legal no município de Pedra do Indaiá, Minas Gerais, Brasil. Legendas: Categoria Trófica (CT), Classe de Frequência (CF). Tipos de registros: Pegadas (P); Registro Fotográfico (RF); Visualização (V); Vocalização (VO). Classes de frequência: Muito Abundante (MA); Abundante (AB); Frequente (FR); Ocasional (OC); Rara (RR); Muito Rara (MR). Categoria de ameaça: Em Perigo (EN); Pouco Preocupante (LC); Vulnerável (VU).

\begin{tabular}{|c|c|c|c|c|c|}
\hline \multirow{2}{*}{ Ordem/Família/Espécie } & \multirow{2}{*}{ Nome comum } & \multirow{2}{*}{ Tipo de registro } & \multirow{2}{*}{$\mathbf{C F}$} & \multicolumn{2}{|c|}{ Categoria de ameaça } \\
\hline & & & & Minas Gerais & Brasil \\
\hline $\begin{array}{l}\text { DIDELPHIMORPHIA } \\
\text { DIDELPHIDAE }\end{array}$ & & & & & \\
\hline $\begin{array}{l}\text { Didelphis albiventris } \\
\text { PILOSA }\end{array}$ & Gambá & $\mathrm{V}, \mathrm{RF}$ & RR & - & - \\
\hline MYRMECOPHAGIDAE & & & & & \\
\hline Myrmecophaga tridactyla & Tamanduá-bandeira & $\mathrm{V}, \mathrm{P}, \mathrm{F}, \mathrm{RF}$ & FR & VU & VU \\
\hline $\begin{array}{l}\text { Tamandua tetradactyla } \\
\text { CINGULATA } \\
\text { DASYPODIDAE }\end{array}$ & Tamanduá-mirim & $\mathrm{RF}$ & MR & - & - \\
\hline
\end{tabular}


Cabassous unicinctus

Dasypus novemcinctus

Euphractus sexcinctus

PRIMATES

\section{CALLITRICHIDAE}

Callithrix penicillata

PITHECIIDAE

Callicebus nigrifrons

CARNIVORA

CANIDAE

Cerdocyon thous

Chrysocyon brachyurus

Lycalopex vetulus

FELIDAE

Leopardus pardalis

Leopardus tigrinus

Puma concolor

Puma yagouaroundi

MEPHITIDAE

Conepatus semistriatus

MUSTELIDAE

Eira barbara

Galictis cuja

Lontra longicaudis

PROCYONIDAE

Nasua nasua

Procyon cancrivorus

LAGOMORPHA

LEPORIDAE

Sylvilagus brasiliensis

RODENTIA

CAVIIDAE

Hydrochoerus hydrochaeris

CUNICULIDAE

Cuniculus paca

\section{ERETHIZONTIDAE}

Coendou prehensilis
Tatu-de-rabo-mole

Tatu-galinha

Tatu-peba

Mico-estrela

Sauá

Cachorro-do-mato

Lobo-guará

Raposinha

Jaguatirica

Gato-do-mato-pequeno

Onça-parda

Gato-mourisco

Jaritataca

Irara

Furão

Lontra

Quati

Mão-pelada

Tapeti

Capivara

Paca

Porco-espinho

$\begin{array}{cc}\text { RF } & \text { RR } \\ \text { V, P, RF } & \text { RR } \\ \text { V, P, RF } & \text { RR }\end{array}$

V, VO

RR

$\mathrm{V}, \mathrm{VO}$

RR

$\begin{array}{cc}\text { V, P, F, RF } & \text { RR } \\ \text { P, F, RF } & \text { OC }\end{array}$

RF

RR

VU

VU

VU

RF

RF

RR

RR

RR

MR

RF

$\mathrm{OC}$

V, P, RF

V, P, RF

RR

RF

RF

RR

MR

$\mathrm{V}, \mathrm{RF}$

RR

$\mathrm{V}, \mathrm{RF}$

RR

RF

RR

P, F, RF

RR

P, RF

RR

RR 
FIGURA 2: Curva de acumulação (observada e estimada por Jackknife de primeira ordem) de espécies de mamíferos de médio e grande porte em uma área de Reserva Legal no município de Pedra do Indaiá, Minas Gerais, Brasil.

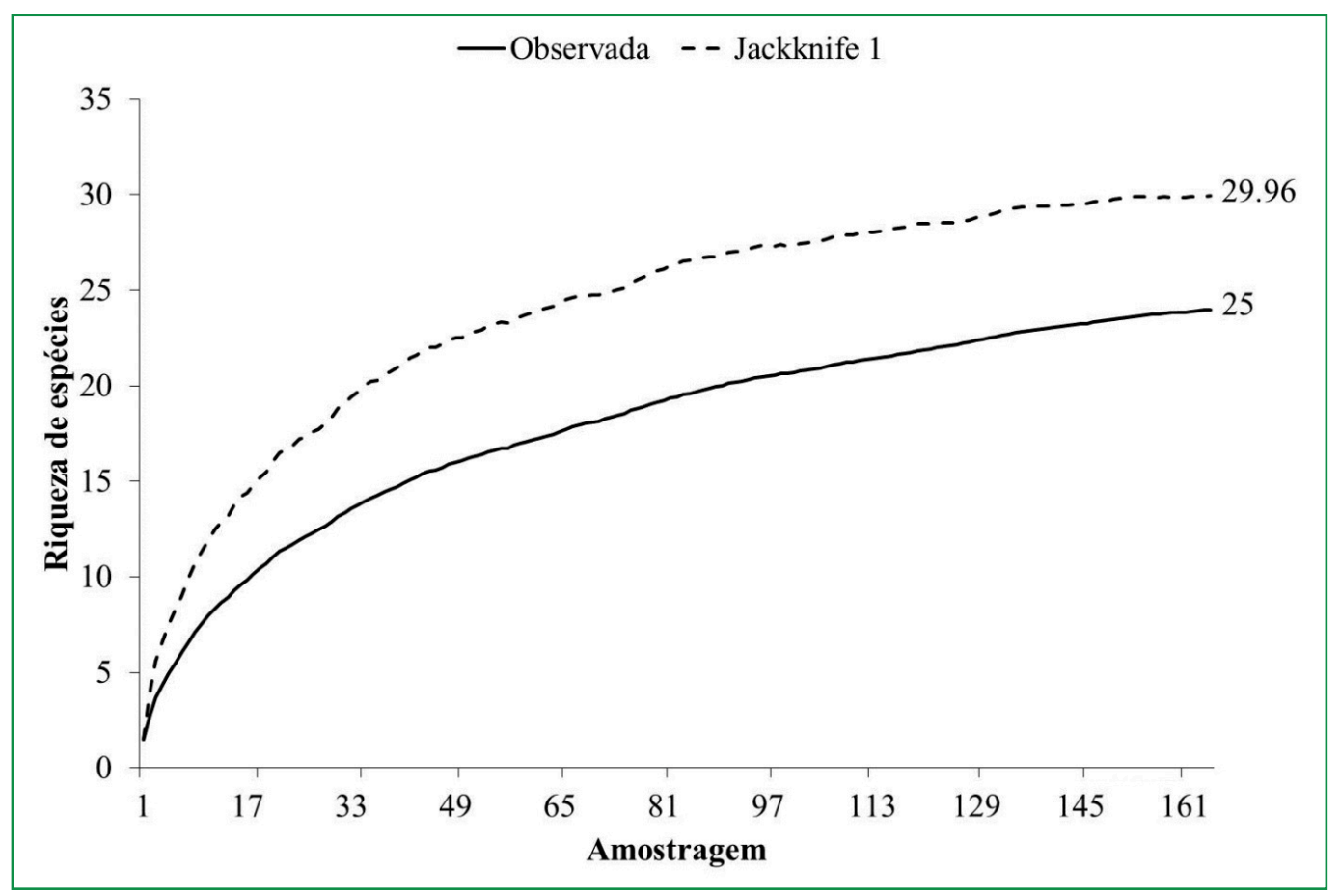

Das 25 espécies registradas na RL, seis encontramse ameaçadas de acordo com a Lista de Espécies Ameaçadas de Extinção da Fauna do Estado de Minas Gerais (DRUMMOND et al., 2008): M. tridactyla (Critério: C2a(i); Figura 3A), C. brachyurus (Critério: A2cde; Figura 3C), Leopardus pardalis (Critério: C2a(i); Figura 3), Leopardus tigrinus (Critério: C2a(i); Figura 3E), Puma concolor (Critério: C2a(i); Figura 3D) e Lontra longicaudis (Critério: A2cde); e quatro se encontram na Lista Nacional Oficial de Espécies da Fauna Ameaçadas de Extinção (MMA, 2014): M. tridactyla (Critério: A2c; MIRANDA et al., 2015), C. brachyurus (Critério: A3c), Lycalopex vetulus (Critério: A $2+3 \mathrm{~cd}$; Figura 3B), L. tigrinus (Critério: C1), P. concolor (Critério: A2bcd $+3 \mathrm{~cd}$; C1) e Puma yagouaroundi (Critério: C1; Figura $3 \mathrm{G})$. Cabe ressaltar que, embora não pertençam à fauna de mamíferos silvestres, foram registradas (através de registro fotográfico e visualização), durante todo o período de estudo, espécies domésticas como Canis lupus familiaris e Bos tauros. 
FIGURA 3: Espécies de mamíferos ameaçados de extinção registrados em uma área de Reserva Legal no município de Pedra do Indaiá, Minas Gerais, Brasil. A: Myrmecophaga tridactyla, B: Lycalopex vetulus, C: Chrysocyon brachyurus, D: Puma concolor, E: Leopardus tigrinus, F: Leopardus pardalis e G: Puma yagouaroundi.
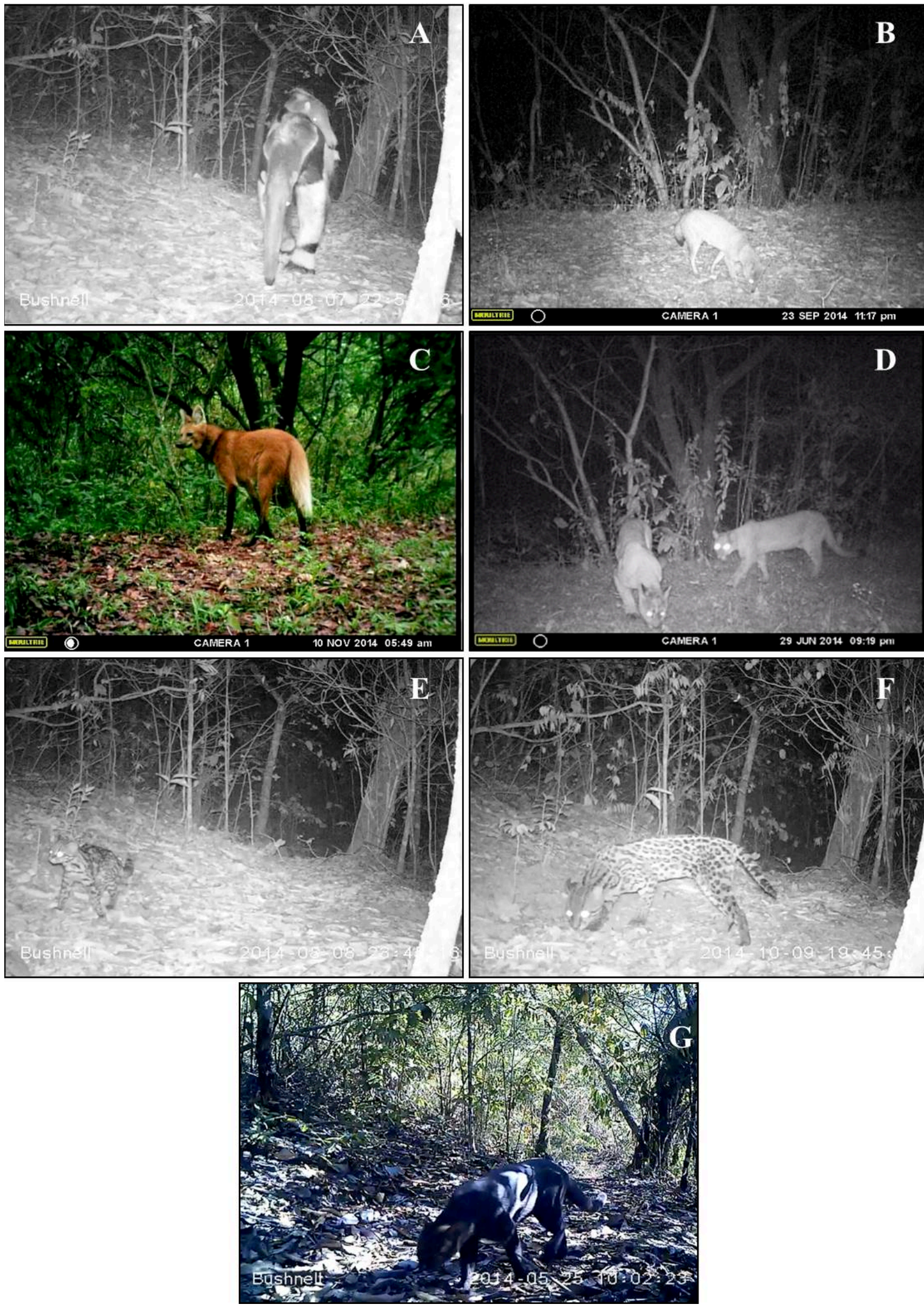


\section{Discussão}

Considerando os padrões encontrados em outras áreas de Cerrado, a riqueza de mamíferos terrestres de médio e grande porte presentes na área de estudo foi elevada (BOCCHIGLIERI et al., 2010; OLIVEIRA, 2010; RIBEIRO; MELO, 2013; SANTIAGO, 2016; MACHADO et al., 2017), representando $16 \%$ das espécies que ocorrem no Cerrado (REIS et al., 2006). Além do mais, o número de espécies encontrado foi próximo aos estudos conduzidos por Rocha e Dalponte (2006) e Oliveira et al. (2009) em unidades de conservação, nos quais foram registradas 26 e 29 espécies, respectivamente.

Por se tratar de uma RL em "sistema de condomínio" (reserva com mais de uma propriedade situada na mesma área, conforme art. 16 da Lei no ${ }^{\circ}$ 12.651/2012), a área é um dos maiores fragmentos de vegetação preservada na região e atualmente tem a maior parte de suas terras tomadas por pastagens e silvicultura (Eucalyptus ssp.). A presença de vegetação característica do Cerrado stricto sensu junto à mata de galeria e área brejosa constitui diferentes ambientes que possibilitam a exploração de uma maior variedade de recursos, principalmente para forrageamento, abrigo, passagem ou descanso das espécies (BARRETO, 1999; SANTOS et al., 2004; PINHO, 2015).

A ordem Carnivora abriga a maior parte das espécies de mamíferos terrestres de médio e grande porte do Brasil (PAGLIA et al., 2012), sendo também a ordem com maior número de representantes em outros estudos realizados no Cerrado (ROCHA; DALPONTE, 2006; OLIVEIRA et al., 2009; BOCCHIGLIERI et al., 2010; LESSA et al., 2012). Tem sido relatado que essa ordem ocorre com mais frequência em paisagens fragmentadas, não apresentando preferência por habitat, uma vez que possuem grande mobilidade e uma capacidade de explorar novos ambientes quando próximas de manchas de vegetação nativa (LYRA-JORGE et al., 2008; 2010; LESSA et al., 2012).

Em ecossistemas tropicais, as curvas de acumulação de espécies raramente estabilizam (SANTOS, 2003). No entanto, o incremento e a provável estabilização da curva não podem ser descartados com a utilização de outros métodos de amostragem específicos para ampliação da amostragem de outros grupos de mamíferos, como redes de neblina para captura de Chiropteros e armadilhas tipo Sherman e Tomahawk para capturas de pequenos mamíferos (grupos que não entraram na amostragem do presente trabalho).

A presença de animais em alguma categoria de ameaça sugere a necessidade de manutenção da RL. A área de estudo juntamente com os remanescentes de vegetação nativa presentes no seu entorno podem auxiliar na manutenção da qualidade do habitat necessária à persistência das populações na região, bem como os serviços ecossistêmicos prestados por ela. Além do mais, a existência de propriedades rurais junto aos limites da RL pode ajudar a explicar a grande frequência de espécies domésticas. A presença de animais domésticos, em especial $C$. familiaris, pode representar uma ameaça à mastofauna local (ROCHA; DALPONTE, 2006; RIBEIRO; MELO, 2013). Essas espécies são consideradas potenciais transmissoras de doenças e têm a capacidade de predar animais silvestres, além de serem competidores diretos por recursos alimentares (ROCHA; DALPONTE, 2006; OLIVEIRA et al., 2009). Cabe ressaltar que, previamente à época de realização desse trabalho, foi encontrado na área do estudo um exemplar de T. tetradactyla morto, com ferimentos ocasionados por cães. Também durante os levantamentos nos transectos foi possível, por três ocasiões, escutar cães latindo nas áreas de mata.

Os resultados obtidos através deste estudo mostraram que a RL investigada, embora apresente uma pequena área quando comparada a outros trabalhos, apresentou uma riqueza considerável de mamíferos terrestres de médio e grande porte. Ainda que este trabalho não tenha considerado a distribuição das espécies na região de estudo, os dados apresentados evidenciam a importância da RL para manutenção da fauna local de mamíferos, podendo contribuir como local de refúgio, alimentação, passagem e/ou descanso. Nesse sentido, tornam-se necessárias novas pesquisas que visem avaliar a dinâmica das espécies entre os fragmentos de RL presentes na região de estudo, evidenciando aqueles com maior importância para a persistência das espécies na paisagem. 


\section{Referências}

ABREU JR, E. F.; KÖHLER, A. Mastofauna de médio e grande porte na RPPN da UNISC, RS, Brasil. Biota Neotropica, Campinas, v. 9, n. 4, p. 169-174, 2009.

ALVES, T. R.; FONSECA, R. C. B.; ENGEL, V. L. Mamíferos de médio e grande porte e sua relação com o mosaico de habitats na cuesta de Botucatu, estado de São Paulo, Brasil. Iheringia Série Zoologia, Porto Alegre, v. 102, n. 2, p. 150-158, 2012.

ANTUNES, A. Z.; ESTON, M. R. Mamíferos (Chordata: Mammalia) florestais de médio e grande porte registrados em Barreiro Rico, Anhembi, estado de São Paulo. Revista do Instituto Florestal, São Paulo, v. 21, n. 2, p. 201-215, 2009.

BARRETO, C. C. Heterogeneidade espacial do habitat e diversidade específica: implicações ecológicas e métodos de mensuração. In: SILVA, S. H. G.; LAVRADO, H. P. (Ed.). Ecologia dos ambientes costeiros do estado do Rio de Janeiro. Série Oecologia brasiliensis. Rio de Janeiro: PPGE-UFRJ, 1999. p. 121-153.

BECKER, M.; DALPONTE, J. C. Rastros de mamíferos silvestres brasileiros: um guia de campo. Rio de Janeiro: Technical Books Editora, 2013. $170 \mathrm{p}$.

BOCCHIGLIERI, A.; MENDONÇA, A. F.; HENRIQUES, R. P. B. Composição e diversidade de mamíferos de médio e grande porte no Cerrado do Brasil central. Biota Neotropica, Campinas, v. 10, n. 3, p. 170-176, 2010.

BORGES, P. A. L.; TOMÁS. W. M. Guia de rastros e outros vestígios de mamíferos do pantanal. Corumbá: Embrapa Pantanal, 2004. 148 p.

BRASIL. Código Florestal. 2012. Disponível em: <http://www. planalto.gov.br/ccivil_03/_ato2011-2014/2012/lei/112651.htm>.

BROCARDO, C. R.; CANDIDO JUNIOR, J. F. Persistência de mamíferos de médio e grande porte em fragmentos de floresta ombrófila mista no estado do Paraná, Brasil. Revista Árvore, Viçosa, v. 36, n. 2, p. 301-310, 2012.

CANEVARI, M.; VACCARO, O. Guía de mamíferos del sur de América del Sur. Buenos Aires: L.O.L.A., 2007. 413 p.

CARDILlO, M.; MACE, G. M.; JONES, K. E.; BIELBY, J.; BININDA-EMONDS, O. R.; SECHREST, W.; ORME, C. D.; PURVIS, A. Multiple causes of high extinction risk in large mammal species. Science, New York, v. 309, n. 5738, p. 1239-1241, 2005.

COLWELL, R. K. EstimateS: statistical estimation of species richness and shared species from samples. User's Guide and application. 2013. Disponível em: <http://purl.oclc.org/estimates>. COPAM - CONSELHO ESTADUAL DE POLÍTICA AMBIENTAL. Lista de espécies ameaçadas de extinção da fauna do estado de Minas Gerais. 2010. Disponível em: <http:// www.siam.mg.gov.br/sla/download.pdf?idNorma=13192>.

COSTA, L. P.; LEITE, Y. L. R.; MENDES, S. L.; DITCHFIELD, A. D. Mammal conservation in Brazil. Conservation Biology, San Francisco, v. 19, n. 3, p. 672-679, 2005.

DOTTA, G.; VERDADE, L. M. Trophic categories in a mammal assemblage: diversity in an agricultural landscape. Biota Neotropica, Campinas, v. 7, n. 2, p. 287-292, 2007.

DRUMMOND, G. M.; MACHADO, A. B. M.; MARTINS, C. S.; MENDONÇA, M. P.; STEHMANN, J. R. Listas vermelhas das espécies da fauna e da flora ameaçada de extinção em Minas Gerais. Belo Horizonte: Fundação Biodiversitas, 2008. 70 p.

DUPRAT, P. L.; ANDRIOLO, A. Mastofauna não-voadora de médio e grande porte em um fragmento de Mata Atlântica no município de Rio Novo, MG. Revista Brasileira de Zoociências, Juiz de Fora, v. 3, n. 1, 2, 3, p. 163-172, 2011.

GALETTI, M.; PARDINI, R.; DUARTE, J. M. B.; SILVA, V. M. F.; ROSSI, A.; PERES, C. A. Mudanças no Código Florestal e seu impacto na ecologia e diversidade dos mamíferos no Brasil. Biota Neotropica, Campinas, v. 10, n. 4, p. 47-52, 2010.

IBGE - INSTITUTO BRASILEIRO DE GEOGRAFIA E ESTATÍSTICA. Pedra do Indaiá. 2010. Disponível em: <http:// cidades.ibge.gov.br/xtras/perfil.php?lang=\&codmun=314890\&sea $\mathrm{rch}=$ minas-gerais $\mid$ pedra-do-indaia $>$.

JOHNSON, C. N.; BALMFORD, A.; BROOK, B. W.; BUETTEL, J. C.; GALETTI, M.; GUANGCHUN, L.; WILMSHURST, J. M. Biodiversity losses and conservation responses in the Anthropocene. Science, New York, v. 356, n. 6335, p. 270-275, 2017.

LESSA, L. G.; ALVES, H.; GEISE, L.; BARRETO, R. M. F. Mammals of medium and large size in a fragmented Cerrado landscape in northeastern Minas Gerais state, Brazil. Check List, Rio Claro, v. 8, n. 2, p. 192-196, 2012.

LINSDALE, J. M.; RODGERS. T. L. Frequency of occurrence of birds in Alum Rock Park, Santa Clara County, California. The Condor, Albuquerque, v. 39, n. 3, p. 108-11, 1937.

LYRA-JORGE, M. C.; CIOCHETI, G.; PIVELLO, V. R. Carnivores mammals in a fragmented landscape in northeast of São Paulo state, Brazil. Biodiversity and Conservation, New York, v. 17, n. 7, p. 1573-1580, 2008.

LYRA-JORGE, M. C.; RIBEIRO, M. C.; CIOCHETI, G.; TAMBOSI, L. R.; PIVELLO, V. R. Influence of multi-scale landscape structure on the occurrence of carnivorous mammals in a human-modified savanna. Brazil. European Journal of Wildlife Research, Berlin, v. 56, n. 3, p. 359-368, 2010.

MACHADO, F. S.; MOURA, A. S.; SANTOS, K. K.; MENDES, P. B.; ABREU, T. C. K.; FONTES, M. A. L. Registros ocasionais de mamíferos de médio e grande porte na microrregião de Lavras e São João del Rei, Campo das Vertentes, Minas Gerais. Revista Agrogeoambiental, Pouso Alegre, v. 9, n. 1, p. 35-44, 2017.

MAZZOLLI, M. Persistência e riqueza de mamíferos focais em sistemas agropecuários no planalto meridional brasileiro. 2006. 105 f. Tese (Doutorado em Ecologia) - Universidade Federal do Rio Grande do Sul, Porto Alegre. 2006.

METZGER, J. P. O Código Florestal tem base científica? Natureza \& Conservação, Curitiba, v. 8, n. 1, p. 1-5, 2010.

MIRANDA, F. R.; CHIARELLO, A. G.; RÖHE, F.; BRAGA, F. G.; MOURÃO, G. M.; MIRANDA, G. H. B.; SILVA, K. F. M.; FARIACORRÊA, M. A.; VAZ, S. M.; BELENTANI, S. C. S. Avaliação do risco de extinção de Myrmecophaga tridactyla Linneus, 1758 no Brasil. In: INSTITUTO CHICO MENDES DE CONSERVAÇÃO DA BIODIVERSIDADE (Ed.). Avaliação do Risco de Extinção de Xenartros brasileiros. Brasília: ICMBio, 2015. p. 89-105.

MMA - MINISTÉRIO DO MEIO AMBIENTE. Lista nacional oficial de espécies da fauna ameaçadas de extinção. 2014. Disponível em: <http://www.in.gov.br/autenticidade.html >. 
MORRISON, J. C.; SECHREST, W.; DINERSTEIN, E.; WILCOVE, D. S.; LAMOREUX, J. F. Persistence of large mammal faunas as indicators of global human impacts. Journal of Mammalogy, Lawrence, v. 88, n. 6, p. 363-1380, 2007.

OLIVEIRA, I. M. Riqueza, abundância de espécies e uso de habitat por mamíferos de médio e grande porte em cinco unidades de conservação no Cerrado. 2010. 91 f. Dissertação (Mestrado em Ecologia) - Universidade de Brasília, Brasília. 2010. OLIVEIRA, T. G.; CASSARO, K. Guia de identificação dos felinos brasileiros. São Paulo: Sociedade de Zoológicos do Brasil, 1999. $60 \mathrm{p}$.

OLIVEIRA, V. B.; CÂMARA, E. M. V. C.; OLIVEIRA, L. C. Composição e caracterização da mastofauna de médio e grande porte do Parque Nacional da Serra do Cipó, Minas Gerais, Brasil. Mastozoología Neotropical, Mendoza, v. 16, n. 2, p. 355-364, 2009.

PAGLIA, A. P.; FONSECA, G. A. B.; RYLANDS, A. B.; HERRMANN, G.; AGUIAR, L. M. S.; CHIARELLO, A. G.; LEITE, Y. L. R.; COSTA, L. P.; SICILIANO, S.; KIERULFF, M. C. M.; MENDES, S. L.; TAVARES, V. C.; MITTERMEIER, R. A.; PATTON, J. L. Lista anotada dos mamíferos do Brasil/ Annotated checklist of Brazilian mammals. 2. ed. Occasional Papers in Conservation Biology, $\mathrm{n}^{-}$6. Arlington: Conservation International, 2012. $76 \mathrm{p}$.

PARDINI, R.; DITT, E. H.; CULLEN JR, L.; BASSI, C.; RUDRAN, R. Levantamento rápido de mamíferos terrestres de médio e grande porte. In: CULLEN, JR. L.; RUDRAN R.; VALLADARES-PÁDUA, C. (Ed.). Métodos de estudo em Biologia da Conservação \& Manejo da Vida Silvestre. Curitiba: Editora da Universidade Federal do Paraná, 2006. p. 181-201.

PINHEIRO, R. S.; BORGES, L. S.; SCHERER, C. S.; OLIVEIRA, T. V.A. mastofauna terrestre da Universidade Federal do Recôncavo da Bahia, Campus Cruz das Almas, Brasil. Magistra, Cruz das Almas, v. 29, n. 1, p. 106-115, 2017.

PINHO, F. F. Influência de fatores ambientais sobre a ocorrência e diversidade de mamíferos de médio e grande porte em unidades de conservação da Serra do Espinhaço Meridional. 2015. 74 f. Dissertação (Mestrado em Ecologia de Biomas Tropicais) - Universidade Federal de Ouro Preto, Ouro Preto. 2015. REIS, N. R.; PERACCHI, A. L.; PEDRO, A. L.; LIMA, A. W. Mamíferos do Brasil. Londrina: Editora da UEL, 2006. 437 p.
RIBEIRO, P.; MELO, F. R. Mamíferos de médio e grande porte de uma área agricultável em Terezópolis (GO) com notas sobre métodos de amostragem. Neotropical Biology and Conservation, São Leopoldo, v. 8, n. 2, p. 68-78, 2013.

ROCHA, E. C.; DALPONTE, J. C. Composição e caracterização da fauna de mamíferos de médio e grande porte em uma pequena reserva de Cerrado em Mato Grosso, Brasil. Revista Árvore, Viçosa, v. 30, n. 4, p. 669-678, 2006.

RORIZ, P. A. C.; FEARNSIDE, P. M. A construção do Código Florestal Brasileiro e as diferentes perspectivas para a proteção das florestas. Novos Cadernos NAEA, Belém, v. 18, n. 2, p. 51-68. 2015.

SANTIAGO, R. Mastofauna de médio e de grande porte da Estação Experimental Syngenta de Uberlândia - MG. Revista Intertox de Toxicologia Risco Ambiental e Sociedade, São Paulo, v. 9, n. 1, p. 132-149, 2016.

SANTOS, A. J. dos. Estimativas de riqueza em espécies. In: CULLEN JR., L.; RUDRAM, R.; VALLADARES-PADUA, C. (Org.). Métodos de estudos em biologia da conservação e manejo da vida silvestre. Curitiba: UFPR/Fundação O Boticáio, 2003. p. 19-41.

SANTOS, M. F. M.; PELLANDA, M.; TOMAZZONI, A. C.; HASENACK, H.; HARTZ, S. M. Mamíferos carnívoros e sua relação com a diversidade de hábitats no Parque Nacional dos Aparados da Serra, sul do Brasil. Iheringia, Porto Alegre, v. 94, n. 3, p. 235-245, 2004.

SANTOS-FILHO, M.; SILVA, M. N. F. Uso de habitat por mamíferos em áreas de Cerrado do Brasil Central: um estudo com armadilhas fotográficas. Revista Brasileira de Zoociências, Juiz de Fora, v. 4, n. 1, p. 57-73, 2002.

SAZIMA, M.; FABIÁN, M. E.; SAZIMA, I. Polinização de Luehea speciosa (Tiliaceae) por Glossophaga soricina (Chiroptera, Phyllostomidae). Revista Brasileira de Biologia, São Carlos, v. 42, n. 3, p. 505-513, 1982.

TONHASCA JR., A. Ecologia e história natural da Mata Atlântica. Rio de Janeiro: Interciência, 2005. 198 p.

ZEE/MG - ZONEAMENTO ECOLÓGICO-ECONÔMICO DE MINAS GERAIS. Shapefile do mapeamento da cobertura vegetal. 2009. Disponível em: $<$ http://geosisemanet.meioambiente. mg.gov.br/zee/>. 